\title{
Pros and Cons of serological testing in syphilis diagnosis and follow up
}

\author{
Gino Ciarrocchi', Marco D'anzeo', Luca Butini², Giuliano Brandozzi ${ }^{3}$, Davide Drenaggi ${ }^{4}$, Massimo Simeone ${ }^{5}$ \\ I S.O. Laboratorio analisi \\ 2 S.O. Clinica di Immunologia \\ 3 S.O. Clinica di Dermatologia \\ 4 S.O. Clinica di Malattie Infettive \\ 5 S.O. Malattie Infettive - A.O. Ospedali Riuniti Policlinico - Ancona
}

Key words: syphilis, serology, elisa, follow up

L’essenziale ed il superfluo nella diagnosi sierologica e nel follow up di sifilide

\section{SUMMARY}

Since a proper diagnosis of syphilis is often difficult due to the wide variability of both clinical picture and laboratory test results, early recognition of infection caused by Treponema pallidum is crucial for a timely and effective treatment. In most cases, definitive diagnosis relies upon serological testing. A screening ELISA test, coupled with a quantitative RPR test and specific IgM antibodies detection, is currently regarded as the basic diagnostic procedure. In addition, a quantitative particle agglutination TP-PA test, FTA-abs IgG test and, eventually, a western-blot IgG and IgM test, allow to achieve a whole serological pattern for each patient at the time of first diagnosis.

In this study, a group of serum samples $(n=107)$ and cerebro-spinal fluid $(n=3)$ were retrospectively analyzed using the above mentioned tests. A population of 19 patients whose clinical picture was unremarkable for syphilis, showed border-line values at screening and negative results on confirmation tests. Thirty-three out of 91 luetic patients were diagnosed as primary or early secondary syphilis, 36 as latent syphilis, 3 as neurosyphilis, and 3 were neonates with passive specific immunization.

Quantitative RPR test and detection of specific IgM antibodies exhibited extremely high values in all 33 primary syphilis patients; a whole positive luetic pattern was also obtained by confirmation tests. Searching for lgM antibodies, a capture elisa test compared with a single device rapid elisa test showed an overall concordance of $98.1 \%$.

In luetic patients other than primary syphilis, quantitative RPR test and detection of specific IgM antibodies provided less relevant values and a low prevalence pattern, whereas TP-PA and FTA-abs tests showed persistent positives results.

In the follow up of 19 initially treated patients, quantitative RPR values and specific IgM antibodies index showed a slow, progressive decrease until negative.

Conclusion: a comprehensive initial sieroluetic framework is a relevant diagnostic tool for primary diagnosis; along the follow up, elisa screening test,TP-PA and FTA-abs IgG have a poor clinical significance; instead, quantitative RPR titers and specific IgM antibody index change over time, and as such, they are reliable tools to evaluate the therapeutic efficacy of treatment and a rational management of luetic patients.

\section{INTRODUZIONE}

La sifilide è una malattia sessualmente trasmessa altamente contagiosa, causata da Treponema pallidum. Una volta penetrata nell’organismo, la spirocheta si diffonde rapidamente entro 48-72 ore per via sistemica, alternando periodi sintomatici ad altri di lunga latenza clinica. Tradizionalmente, il decorso del'infezione viene classificato in differenti stadi: primario, secondario, latente (precoce e tardivo) e terziario, in relazione agli specifici indizi clinico-anamnestici $(8,20)$.

Dopo circa 2-3 settimane dal contatto, il battere causa la comparsa di una lesione genitale (ulcera primaria o cancro) non dolente, non purulenta e indurita a cui si accompagna comunemente una linfoadenopatia regionale $(2,8,20)$. La fase primaria ha una durata di circa 8-12 settimane, ed è seguita dalla scomparsa della sintomatologia. La risposta anticorpale all'infezione si evidenzia con il riscontro di IgM specifiche dopo circa 2-3 settimane, a cui fa seguito entro 4-6 settimane la produzione di IgG; queste ultime rimarranno presenti in circolo per lunghissimo tempo, finanche per l'intera vita del paziente.

La diagnosi di sifilide è spesso difficoltosa, sia a causa del variabile quadro clinico iniziale che del peculiare andamento dell'infezione. Ad esempio, solo un terzo dei casi si presenta con tutti i caratteri patognomonici; più frequentemente la lesione singola iniziale è assente o nascosta alla visualizzazione, specialmente nelle donne ed in soggetti omosessuali maschi, contribuendo all'instaurarsi di una sifilide "non riconosciuta”, con potenziali sequele cliniche e ricadute sociali ed epidemiologiche (20).

Una sifilide non diagnosticata ha tendenza alla guarigione solo nel $25 \%$ dei casi (20); più spesso, la diffusione sistemi- ca della spirocheta (fase secondaria) causa manifestazioni maculo-papulose dermatologiche e mucose, riscontrabili entro i 3-6 mesi, a cui segue negli anni un lungo periodo di latenza clinica. La fase terziaria può insorgere invece anche a distanza di alcune decadi con manifestazioni patologiche a carico di vari organi (pareti arteriose, cuore, sistema nervoso, apparato osseo) $(1,20)$. Infine, non va dimenticata un’altra potenziale sequela, costituita da casi di sifilide congenita in neonati con madre luetica non trattata o misconosciuta $(7,12,19)$.

Dunque, il precoce riconoscimento dell'infezione da T. pallidum ha un'importanza fondamentale per un corretto e tempestivo intervento terapeutico.

In quadri clinici così sfumati ("lue decapitata”), l’ipotesi diagnostica si potrà basare sui soli dati anamnestici (contatti sessuali a rischio), ma si potrà avvalere fondatamente dei risultati forniti dal vasto arsenale di test di laboratorio, alcuni dei quali sono in grado di rivelare precocemente marcatori sierologici del contatto con T. pallidum (16).

Se storicamente il primo approccio diagnostico avveniva mediante l'indaginoso test di Wasserman e la ricerca di reagine non specifiche, con risultati poco riproducibili $(2,3,6$,$) ,$ l'evoluzione delle conoscenze immunopatogenetiche e la disponibilità di tecnologie innovative hanno permesso in gran parte dei laboratori la diffusione di test standardizzati ed automatizzabili, quali, per solo citare i più impiegati, la tecnica elisa o quella a chemiluminescenza, nelle loro varianti (13), con un corollario di altri test tra cui quello non treponemico, VDRL (o la sua variante RPR), congiuntamente alla ricerca di anticorpi specifici IgM anti-T. pallidum. Sarà evidenziato più avanti il rilievo assunto dai due test anche nel

\section{Corresponding author: Gino Ciarrocchi}

S.O. Laboratorio analisi, S.O.S. Sierologia, A.O. Ospedali Riuniti Policlinico - Ancona

Tel. 07I-596425I - Fax 07I-5964638

E-mail: ciarrokki@libero.it 
percorso diagnostico post-terapeutico. Il test VDRL non è specifico dell'infezione ed è inficiato da numerose false positività biologiche e tecniche; tuttavia esso riveste tuttora un ruolo non trascurabile nella sierodiagnosi luetica $(6,8,11,15,16)$.

Viceversa la ricerca di anticorpi IgM anti-T.pallidum, effettuata con metodologie semplici ed efficaci, aggiunge un elemento di specificità nella fase diagnostica iniziale, e grande rilevanza nel follow up $(11,15)$. Ulteriori test, quali il test quantitativo di agglutinazione TP-PA, quello a fluorescenza indiretta FTA-abs IgG, fino all'impiego della tecnica Western-Blot IgG e IgM, delineano un pattern sieroluetico iniziale, che, insieme agli indizi clinico-anamnestici, costituirà il presidio diagnostico di riferimento per ogni decisione clinica e per l'eventuale monitoraggio sierologico post-terapeutico $(3,4,5,18)$.

L'impiego di un così vasto arsenale sierodiagnostico si giustifica con la constatazione che, a tutt'oggi, non esiste un vero "marker" di guarigione clinica del paziente luetico, da porre in stretta associazione con l'efficacia del trattamento antibiotico.

In tali considerazioni sono compresi, sebbene con diverse motivazioni, i pur preziosi test di amplificazione genica, impiegati a scopo diagnostico per la ricerca del DNA di T.pallidum in campioni biologici, sia nella fase precoce dell'infezione che in quelle tardive o latenti $(9,10,14,16,17)$.

\section{SCOPO}

Valutare il significato della ricerca di IgM anti-T.pallidum e della determinazione quantitativa del titolo anticorpale RPR in soggetti con sospetto di lue primaria, e nelle differenti fasi dell'infezione, e il loro andamento durante il decorso postterapeutico fino alla negativizzazione, quali indicatori dell'efficacia del trattamento terapeutico. Verificare la concordanza e l'efficienza diagnostica di due test elisa IgM antiT.pallidum nel caratterizzare il quadro sierologico in differenti gruppi di pazienti sieroluetici.

\section{MATERIALI E METODI}

Un totale di 110 campioni di siero (107) e liquor cefalo-rachidiano (3), appartenenti a soggetti adulti e pediatrici, con quadro siero-luetico iniziale positivo o con generico sospetto di sifilide fu retrospettivamente selezionato ed studiato mediante impiego di esami sierologici.

La diagnosi clinica fu formulata secondo i consueti criteri clinici-anamnestici, ovunque descritti (20). I gruppi di pazienti furono così suddivisi: lue primaria $(\mathrm{n}=33)$, lue secondaria $(n=16)$, lue latente $(n=36)$, neurosifilide $(n=3)$, immunizzazione passiva neonatale $(n=3)$, sospetto generico di lue $(n=19)$. I sieri risultati positivi al solo test di screening, non confermati con test di secondo livello e privi di indizi clinico-anamnestici furono considerati come campioni falsi positivi. Un totale di 19 pazienti furono seguiti nel follow-up post terapeutico per osservare l'andamento di ogni singolo test.

I campioni furono esaminati con lo scopo di delineare il pattern sieroluetico completo, relativo allo stato dell'infezione di ogni singolo paziente. Lo screening dei campioni fu effettuato con un test ELISA a competizione “one step”, eseguito su analizzatore automatico. La ricerca di IgM anti-T.pallidum fu effettuata mediante confronto tra due metodi ELISA: il primo con impiego di un singolo "device” per campione, ed esecuzione automatizzata rapida; il secondo, nella versione detta "a cattura"; ad entrambi fu aggiunto quale riferimento un test con tecnica Western-Blot. Quale esame non treponemico fu impiegato il test RPR, eseguito con quantizzazione del titolo anticorpale.

Per lo studio furono impiegati i seguenti reagenti forniti in kit commerciali completi:

- Pathozyme Syphilis competition; ii) Immutrep RPR;

- Pathozyme Syphilis M capture, prodotti da Omega Diagnostics, UK (ALIFAX - Italia);

- Serodia TP-PA - Fujirebio, Japan (Alfa Wasserman - Italia);
- T.pallidum IgG IFA assay - Alphadia, Belgio (Alifax - Italia); - T.pallidum IgM Chorus e Fast-Syphilis - Diesse Diagnostics, Italia;

- T.pallidum IgM MarBlot - Trinity Diagnostics, USA (Alifax - Italia).

\section{RISULTATI}

Dei 110 campioni esaminati, 19 di essi risultarono con valori "border-line" al test di screening, negatività a tutti i test di conferma ed assenza di indizi clinici e anamnestici significativi (Tabella 1).

Tutti gli altri 91 campioni, risultati positivi al test Lue-screen, furono sottoposti ai test di conferma per completare il pattern sieroluetico.

I 33 sieri con Lue $\mathrm{I}^{\circ}$ risultarono positivi sia al test Lue-screen che agli altri test di conferma; in particolare, RPR mostrò titoli elevatissimi, confermati altresì dai due test IgM ;

nei 16 sieri con Lue $\mathrm{II}^{\circ}$ il test RPR risultò positivo in 15/16; IgM Chorus in 6/16; IgM Omega in 6/16; i test TP-PA e FTAabs risultavano ugualmente positivi a vario titolo (Tabella 2).

Dei 36 campioni con Lue latente, 24 erano positivi al test RPR; 3 positivi al test IgM Chorus; 5 positivi al test IgM Omega,

I 3 campioni con neurosifilide risultarono positivi al test RPR; allo stesso test, i 3 campioni di neonati con immunizzazione passiva furono negativi; tutti i 6 campioni mostrarono indici negativi di IgM (Tabella 3).

Nella Tabella 4 è mostrato il confronto tra i due test IgM: dei 110 campioni esaminati: 47 risultarono concordemente positivi o border-line, 61 negativi, 2 furono discordanti. All'ulteriore esame con il test Western-Blot IgM entrambi risultarono negativi. La concordanza totale tra i due test ELISA fu del 98.1\%.

Nella Tabella 5 è illustrato lo stesso confronto ristretto a 43 sieri luetici, con l'aggiunta del test Western-Blot IgM. La concordanza totale verso quest'ultimo test fu $97.6 \%$ per IgM Chorus e 93\% per IgM Omega.

La Tabella 6 illustra l'andamento quantitativo dei titoli anticorpali rilevati dai test RPR e IgM Chorus nel follow up posttrattamento di 19 pazienti, definiti inizialmente con sifilide primaria o secondaria precoce. Tutti i 19 pazienti erano positivi ad entrambi i test con indici elevatissimi sia al momento della diagnosi iniziale che dopo 4 mesi dal trattamento terapeutico. Nei successivi controlli, gli indici di positività di IgM decrescevano significativamente fino a diventare negativi in 11/16 pazienti dopo 5-18 mesi (mediana $=11.5$ ). Il titolo anticorpale di RPR divenne negativo in 10 pazienti entro 5-18 mesi (mediana $=13.2)$.

Infine, la Tabella 7 mostra i risultati del test rapido SyphilisFast in 145 soggetti suddivisi in gruppi di pazienti luetici (95) e di soggetti non luetici (50). In quest'ultima popolazione la specificità fu $100 \%$; la sensibilità nei gruppi luetici fu $98.9 \%$, con un'unica eccezione di falsa negatività causata dal fenomeno di pro-zona da eccesso anticorpale, dimostratasi successivamente positiva al test su siero diluito.

\section{DISCUSSIONE}

L'impronta sierologica che caratterizza un soggetto con infezione da T.pallidum costituisce l'evidenza specifica più rilevante dell'infezione nel momento della diagnosi iniziale (lue primaria), superando in specificità anche i segni obbiettivi patognomonici, i quali, sebbene precoci, non sempre corredano il quadro clinico. Il "pattern sieroluetico" che connota ogni singolo paziente è il risultato della specifica reazione immunologica, e su di esso il clinico misurerà la fondatezza dell'ipotesi diagnostica. Osserviamo ora le maggiori evidenze ricavate dallo studio, con il fine di metterne in luce gli aspetti essenziali.

È necessario sottolineare anzitutto che il percorso diagnostico debba avere inizio con un test di screening immunometrico, riproducibile e confrontabile tra differenti laboratori, seguito dal corollario dei test di conferma, qualora si ottenga un primo risultato positivo. L'insieme dei test impiegati delinea in tal modo un preciso e caratteristico quadro sierologi- 
co, facilmente interpretabile per la dimostrazione del contatto con la spirocheta.

Viceversa, pazienti con valori di screening "border-line” e test di conferma negativi, senza indizi clinico-anamnestici di lue, sono da considerare come soggetti con false positività biologiche.

La distinzione tra i quadri sierologici di questi ultimi e i pazienti sieroluetici, nelle varie fasi infettive, risulta in tal modo inequivocabile. Per raggiungere tale certezza, quindi, è necessario eseguire un insieme completo di test e valutarne le specifiche caratteristiche.

Una seconda evidenza risulta dall'impiego dei test Lue-screen, TP-PA, FTA-abs, i quali contribuiscono inizialmente in vario grado a inquadrare correttamente i pazienti luetici; tuttavia, essi forniscono risultati positivi anche nelle fasi post-terapeutiche, con lento e modesto decremento tardivo. Ciò significa che nel follow up questi parametri diagnostici non sono in grado di valutare l'efficacia del trattamento antibiotico; in tale contesto, quindi, forniscono dati superflui allo scopo.

Ciò non avviene invece per i test RPR e IgM anti- T.pallidum, i quali, con caratteristiche diverse, contribuiscono chiaramente a comprendere l'andamento dell'infezione. Ciò consiglia perciò di eseguirli con dosaggio quantitativo fino al momento della loro negativizzazione.

Partendo dal gruppo di pazienti con lue primaria o secondaria precoce, si osserva che tutti i soggetti sono connotati da elevatissimi titoli anticorpali al test RPR (da 1:64 a >1:128), ben al di sopra del titolo di 1:16, considerato in molti studi come un indice diagnostico (11). A ciò fanno da riscontro altrettanto rilevanti indici di IgM anti-T.pallidum. Su quest'ultimo "marker" luetico sono state espresse nel tempo le considerazioni più controverse, legate principalmente a metodologie analitiche indaginose o di modesta efficienza diagnostica $(3,16)$. Nel nostro studio entrambi i metodi impiegati sono innovativi, standardizzati e automatizzabili. Il test rapido IgM Chorus ha una qualità intrinseca basata sull'impiego di selezionati antigeni ricombinanti di Treponema pallidum, perfettamente adattati al dispositivo. Impiegando un singolo "device" monotest, l'esame del campione può essere eseguito in automazione completa in pochi minuti; ciò lo rende oltremodo funzionale per un impiego immediato o urgente.

La concordanza diagnostica tra i due test IgM, dimostrata con confronto verso un test Western-blot, è risultata molto soddisfacente; ciò segna un fondamentale miglioramento diagnostico rispetto alle indaginose tecniche in precedenza impiegate.
Analizzando i risultati ottenuti negli altri gruppi di pazienti luetici, risulta evidente che i due test IgM decrescono seppure lentamente verso una negativizzazione, o residuando non significativi indici di positività entro un ampio arco di tempo, variabile da 5 a 18 mesi. Tale andamento è bene illustrato nel campione di pazienti esaminati durante il follow up post-terapeutico. Di ciò si dovrà tenere conto nel valutare gli indici quantitativi di RPR e IgM, secondo un confronto relativo ai valori precedenti, piuttosto che considerare i singoli valori in modo assoluto.

Infine, un'attenzione particolare deve essere dedicata all'impiego dei test rapidi, impiegati in molti laboratori con criteri talora superficiali. Se è vero che necessità diagnostiche o organizzative (espianto d'organi) inducono talvolta il laboratorio al loro uso per un report immediato, è altrettanto diffuso un impiego incongruo, di un singolo test non specifico, quale RPR, con cui si intende definire un quadro sierololuetico. Lo studio ha dimostrato, viceversa, che in tal caso è discriminante l'impiego di un test rapido treponemico, nel nostro caso Syphilis-Fast, definibile come un test TPHA modificato, per ottenere immediati e specifici indizi diagnostici, anche in assenza di dati clinico-anamnestici.

\section{CONCLUSIONI}

In conclusione, accanto ad un affidabile test di screening, appare essenziale la ricerca quantitativa di IgM anti-T.pallidum e di titoli anticorpali di RPR per definire un quadro sieroluetico di riferimento nel primo approccio diagnostico e, successivamente, nelle varie fasi dell'infezione, dopo il trattamento antibiotico. Gli altri test, richiesti ed eseguiti a corollario, sono utili nella prima formulazione diagnostica, ma pressoché superflui nel decorso post-terapeutico. La ricerca di IgM , in particolare, costituendo l'unico indizio specifico d'infezione luetica variabile con la terapia, deve essere considerata un presidio imprescindibile. Alla luce delle considerazioni avanzate, lo studio ha mostrato come sia possibile valutare e distinguere il ruolo diagnostico di ogni singolo test dell'ampio arsenale analitico. Ciò renderà certamente più agevole il compito del clinico nella gestione del paziente luetico e, non secondario, consentire una scelta razionale ed economica dei soli test con elevato contenuto diagnostico.

Ringraziamenti: ai tecnici di laboratorio Sigg. Giuseppe Rondello, Donatella Pulita, Vinicio Murri per l'alta professionalità e la quotidiana collaborazione.

Tabella I. Totale campioni esaminati con sospetto o diagnosi di LUE

\begin{tabular}{lccc}
\hline Totale campioni $\mathbf{N}=\mathbf{I}$ I0 & LUE-eia screening & Test di conferma positivi & Test di conferma negativi \\
\hline positivo & 91 & 91 & 0 \\
\hline border-line/negativo & 19 & 0 & 19 \\
\hline
\end{tabular}

Tabella 2. Pattern sieroluetico in 33 soggetti con LUE I ${ }^{\mathrm{a}}$ e in 16 soggetti con LUE $/{ }^{\mathrm{a}}$

\begin{tabular}{lccccccc}
\hline & RPR & $\begin{array}{c}\text { IgM-eia } \\
\text { Omega }\end{array}$ & $\begin{array}{c}\text { IgM-eia } \\
\text { Chorus }\end{array}$ & $\begin{array}{c}\text { Lue-eia } \\
\text { screening }\end{array}$ & TP-PA & $\begin{array}{c}\text { FTA-abs } \\
\text { IgG }\end{array}$ \\
\hline LUE I & Pos & 33 & 33 & 33 & 33 & 33 & 33 \\
$(\mathrm{n}=33)$ & Neg & 0 & 0 & 0 & 0 & 0 & 0 \\
\hline LUE II & Pos & 15 & 6 & 6 & 16 & 16 & 16 \\
$(\mathrm{n}=16)$ & Neg & 1 & 10 & 10 & 0 & 0 & 0 \\
\hline
\end{tabular}

Tabella 3. Pattern sieroluetico in 36 soggetti con Lue latente, in 3 soggetti con NeuroLue (3) e in 3 neonati con Immunizzazione passiva neonatale(3)

\begin{tabular}{|c|c|c|c|c|c|c|c|}
\hline & & RPR & $\begin{array}{l}\text { IgM-eia } \\
\text { Omega }\end{array}$ & IgM-Chorus & $\begin{array}{l}\text { Lue-eia } \\
\text { screening }\end{array}$ & TP-PA & $\begin{array}{c}\text { FTA-abs } \\
\text { IgG }\end{array}$ \\
\hline \multirow{2}{*}{$\begin{array}{l}\text { LUE latente } \\
(\mathrm{n}=36)\end{array}$} & Pos & 24 & 5 & 3 & 36 & 36 & 31 \\
\hline & $\overline{\mathrm{Neg}}$ & 12 & 31 & 33 & 0 & 0 & 5 \\
\hline \multirow{2}{*}{$\begin{array}{l}\text { NeuroLUE } \\
(\mathrm{n}=3)\end{array}$} & Pos & 3 & 0 & 0 & 3 & 3 & 3 \\
\hline & $\overline{\mathrm{Neg}}$ & 0 & 3 & 3 & 0 & 0 & 0 \\
\hline \multirow{2}{*}{$\begin{array}{l}\text { Immun.passiva } \\
(\mathrm{n}=3)\end{array}$} & Pos & 0 & 0 & 0 & 3 & 3 & 3 \\
\hline & $\overline{N e g}$ & 3 & 3 & 3 & 0 & 0 & 0 \\
\hline
\end{tabular}


Tabella 4. Confronto tra test elisa IgM CHORUS e IgM capture OMEGA

\begin{tabular}{lcc}
\hline & IgM-OMEGA (Pos + B.L.) & IgM-OMEGA (Negativo) \\
\hline IgM-CHORUS (Pos + B.L) & 47 & 0 \\
\hline IgM-CHORUS (Negativo) & 2 & $6 \mid$
\end{tabular}

Concordanza totale $=108 / 110=98,1 \%$

Tabella 5. Confronto tra IgM CHORUS, IgM OMEGA, IgM W.B. in 43 sieri luetici

\begin{tabular}{lcccc}
\hline & IgM-CHORUS & IgM-OMEGA & IgM-W.B. \\
\hline POS & 27 & $27+2$ & 27 \\
\hline NEG & 10 & 8 & I0 \\
\hline B.L. & 6 & 6 & $3=$ border-line \\
& & & positivo \\
& & & Indeterminato \\
\hline
\end{tabular}

Concordanza Chorus IgM vs W.B.IgM $=42 / 43$ (97,6\%)

Concordanza Omega IgM vs WB IgM $=40 / 43$ (93\%)

Tabella 6. Evoluzione dei titoli di RPR e IgM anti-T.pallidum nella diagnosi iniziale ( 19 pazienti) e nel follow-up fino a 4 mesi ( 19 pazienti) e fino a 18 mesi (I 6 pazienti)

\begin{tabular}{|c|c|c|c|c|}
\hline Tempo dalla diagnosi & $\begin{array}{c}\text { RPR } \\
\text { (titolo anticorpale) }\end{array}$ & $\mathrm{N}^{\circ}$ pazienti & $\begin{array}{c}\text { IgM } \\
\text { (indice anticorpale) }\end{array}$ & $\mathrm{N}^{\circ}$ pazienti \\
\hline \multirow[t]{3}{*}{ Iniziale } & $>=1: 128$ & 15 & Positivo I,2-7,0 & 19 \\
\hline & $\mathrm{I}: 64$ & 4 & Border-line 0,9-I,I & 0 \\
\hline & negativo & 0 & Negativo $<0,9$ & 0 \\
\hline \multirow[t]{7}{*}{ Dopo 4 mesi } & $>=1: 64$ & 15 & $>5,0$ & $\mathrm{I}$ \\
\hline & $\mathrm{I}: 64$ & 2 & $4, I-5,0$ & 0 \\
\hline & $\mathrm{I}: 32$ & 0 & $3,1-4,0$ & $\mathrm{I}$ \\
\hline & $\mathrm{I}: 16$ & 1 & $2, I-3,0$ & 4 \\
\hline & $\mathrm{I}: 8$ & 0 & $\mathrm{I}, 2-2,0$ & 7 \\
\hline & $1: 4$ & $\mathrm{I}$ & $0,9-I, I$ & 5 \\
\hline & Negativo $(<1: 2)$ & 0 & negativo & I \\
\hline \multirow[t]{6}{*}{ Dopo 5-I 8 mesi } & 1.32 & 1 & Positivo I,2-7,0 & $\mathrm{I}$ \\
\hline & $1: 16$ & $\mathrm{I}$ & Border-line 0,9-I,I & 4 \\
\hline & $\mathrm{I}: 8$ & $\mathrm{I}$ & Negativo $<0,9$ & II \\
\hline & $\mathrm{I}: 4$ & 1 & & \\
\hline & $\mathrm{I}: 2$ & 2 & & \\
\hline & negativo & 10 & & \\
\hline
\end{tabular}

Tabella 7. Risultati di Syphilis-FAST in un totale di 145 soggetti: non luetici (50) e luetici (95)

\begin{tabular}{ccc}
\hline & $\begin{array}{c}\text { FAST - SYPHILIS } \\
\text { positivo }\end{array}$ & negativo \\
\hline $\begin{array}{c}\text { LUE IgM positivo } \\
\mathrm{N}=28\end{array}$ & 27 & 0 \\
\hline $\begin{array}{c}\text { LUE IgM border-line } \\
\mathrm{N}=3\end{array}$ & 3 & 0 \\
\hline $\begin{array}{c}\text { LUE IgM negativo } \\
\mathrm{N}=14\end{array}$ & 14 & 0 \\
\hline $\begin{array}{c}\text { LUE-screen pos/border-line } \\
\mathrm{N}=50\end{array}$ & 50 & 50 \\
\hline $\begin{array}{c}\text { Non luetici } \\
\mathrm{N}=50\end{array}$ & 0 &
\end{tabular}

\section{BIBLIOGRAFIA}

1. Castro R, Prieto ES, Aguas MJ, et al. Evaluation of the Treponema pallidum particle agglutination technique (TP-PA) in the diagnosis of neurosyphilis. J Clin Lab Anal 2006; 20(6): 233-8.

2. Centers for Disease Control and Prevention. Sexually transmitted diseases treatment guidelines 2002. MMWR 2002; 51: 1-80.

3. Cole MJ, Perry KR, Parry JV. Comparative evaluation of 15 serological assays for the detection of syphilis infection. Eur J Clin Microbiol Infect Dis 2007 Oct; 26(10): 705-13.

4. Creegan L, Bauer HM, Samuel MC, Klausner J, Liska S, Bolan G. An evaluation of the relative sensitivities of the venereal disease research laboratory test and the treponema pallidum particle agglutination test amomg patients diagnosed with primary syphilis. Sex Transm Dis 2007 Dec; 34(12): 1016-8.

5. Fujimori C, Yukimasa N, Miura K, et al. Evaluation of the anti-TP antibody latex agglutination immunoassay in routine testing and a clinical viewpoint. Rinsho Byori 2009 mar; 57(3): 206-12.

6. Gesau A, Kittler H, Hein U, Dangl-Erlach E, Stingl G, Tschachler E. Biological false-positive tests comprise a high proportion of Venereal Disease Research Laboratory reactions in an analysis of 300,000 sera. Int J STD AIDS 2005 Nov; 16(11): 722-6.

7. Goh BT, Thornton AC. Antenatal screening for syphilis. SEX Transm Infect 2007; 83: 345-6.

8. Kingston M, French P, Goh B, et al. UK National Guidelines on the management of Syphilis 2008. Int J STD AIDS 2008; 19: 729-40.

9. Koek AG, Bruisten SM, Dierdorp M, van Dam AP, Templeton K. Specific and sensitive diagnosis of syphilis using a real-time PCR for Treponema pallidum. Clin Microbiol Infect Dis 2006; 12: 1221-40.

10. Liu H, Rodes B, Chen CY, Steiner B. New tests for syphilis: rational design of a PCR method for detection of Treponema pallidum in clinical specimens using unique regions of the DNA polymerase I gene. $J$ Clin Microbiol 2001; 39: 1941-6.

11. ManaviK, Young H, McMillan A. The sensibility of syphilis assays in 
detecting different stages of early syphilis. Int J STD AIDS 2006 Nov; 17(11): 768-71.

12. Marangoni A, Moroni A, Tridapalli E, et al. Antenatal syphilis serology in pregnant women and follow-up of their infants in northern Italy. Clin Microbiol Infect 2008; 14: 1065-8.

13. Marangoni A, Sambri V, Accardo S, et al. Evaluation of LIAISON® Treponema Screen, a novel recombinant antigen-based chemiluminescence immunoassay for the laboratori diagnosis of syphilis. Clin Diagn lab Immunol 2005; 12: 1231-4.

14. Marfin AA, Liu H, Sutton MY, Steiner B, Pillay A, Markowitz LE. Amplification of the DNA polymerase I gene of Treponema pallidum from whole blood of persons with syphilis. Diagn Microbiol Infect Dis 2001; 40: 163-6.

15. McMillan A, Young H. Qualitative and quantitative aspects of the serological diagnosis of early syphilis. Int J STD AIDS 2008; 19(9): 620-4.

16. Müller I, Brade V, Hagedorn HJ, et al. Is serological testing a reliable tool in laboratory diagnosis of syphilis? Meta-analysis of eight external quality control surveys performed by the German Infection Serology Proficiency Testing Program. J Clin Microbiol 2006; 44(4): 1335-41.

17. Noordhoek GT, Wolters EC, de Jonge ME, Embden JD. Detection by polymerase chain reaction of Treponema pallidum DNA in cerebrospinal fluid from neurosyphilis patients before and after antibiotic treatment. $J$ Clin Microbiol 1991; 29: 1976-84.

18. Palmer HM, Higgins SP, Herring AJ, Kingston MA. Use of PCR in the diagnosis of early syphilis in the United Kingdom. Sex Transm Infect 2003; 79: 479-83.

19. Sambri V, Marangoni A, Eyer C, et al. Western immunoblotting with five Treponema pallidum recombinant antigens for serological diagnosis of syphilis. Clin Diagn Lab Immunol 2001; 8: 534-9.

20. Simms I, Ward H. Congenital syphilis in the United Kingdom. Sex Transm Infect 2006; 82: 1.

21. Zetola NM, Engelman J, Jensen TP, Klausner JD. Syphilis in the United States: an update for clinicians with an emphasis on HIV coinfection. Mayo Clin Proc 2007; 82(9): 1091-102. 